\title{
LA-UR $95-1641$
}

\section{TITLE: TRANSIMS: TRANSPORTATION ANALYSIS AND SIMULATION SYSTEM}

\author{
AUTHOR(S): IARON SMITH, TSA-DO/SA \\ RICHARD BECKMAN, TSA-1 \\ KEITH BAGGERLY, TSA-1 \\ DOUG ANSON, TSA-5 \\ MICHAEL WILIIAMS, TSA-4
}

SUBMITTED TO:

\section{DISCLAIMER}

This report was prepared as an account of work sponsored by an agency of the United States Government. Neither the United States Government nor any agency thereof, nor any of their employees, makes any warranty, express or implied, or assumes any legal liability or responsibility for the accuracy, completeness, or usefulness of any information, apparatus, product, or process disclosed, or represents that its use would not infringe privately owned rights. Reference herein to any specific commercial product, process, or service by trade name, trademark, manufacturer, or otherwise does not necessarily constitute or imply its endorsement, recommendation, or favoring by the United States Government or any agency thereof. The views and opinions of authors expressed herein do not necessarily state or reflect those of the United States Government or any agency thereof. 


\section{DISCLAIMER}

Portions of this document may be illegible in electronic image products. Images are produced from the best available original document. 
TRANSIMS:

\title{
TRansportation ANalysis and SIMulation System
}

\author{
LaRon Smith, Richard Beckman, Keith Baggerly, Doug Anson, Michael Williams \\ Los Alamos National Laboratory
}

\begin{abstract}
This document summarizes the TRansportation ANalysis and SIMulation System (TRANSIMS) Project, the system's major modules, and the project's near-term plans. TRANSIMS will employ advanced computational and analytical techniques to create an integrated regional transportation systems analysis environment. The simulation environment will include a regional population of individual travelers and freight loads with travel activities and plans, whose individual interactions will be simulated on the transportation system, and whose environmental impact will be determined. We will develop an interim operational capability (IOC) for each major TRANSIMS module during the fiveyear program. When the $I O C$ is ready, we will complete a specific case study to confirm the IOC features, applicability, and readiness.
\end{abstract}

\section{Introduction}

The TRansportation ANalysis and SIMulation System (TRANSIMS) is part of the multi-track Travel Model Improvement Program sponsored by the U.S. Department of Transportation and the Environmental Protection Agency. Los Alamos National Laboratory is leading its development. TRANSIMS will address issues resulting from the Intermodal Surface Transportation and Efficiency Act of 1991 , such as considerations of land use policies, intermodal connectivity, and enhanced transit service. It will support analyses of potential responses to the stringent air-quality requirements of the Clear Air Act Amendments of 1990.

The TRANSIMS Project objective is to develop a set of mutually supporting realistic simulations, models, and data bases that employ advanced computational and analytical techniques to create an integrated regional transportation systems analysis environment. By applying forefront technologies and methods, it will simulate the dynamic details that contribute to the complexity inherent in today's and tomorrow's transportation issues. The integrated results from the detailed simulations will support transportation planners, engineers, and others who must address environmental pollution, energy consumption, traffic congestion, land use planning, traffic safety, intelligent vehicle efficacies, and the transportation infrastructure effect on the quality of life, productivity, and economy.

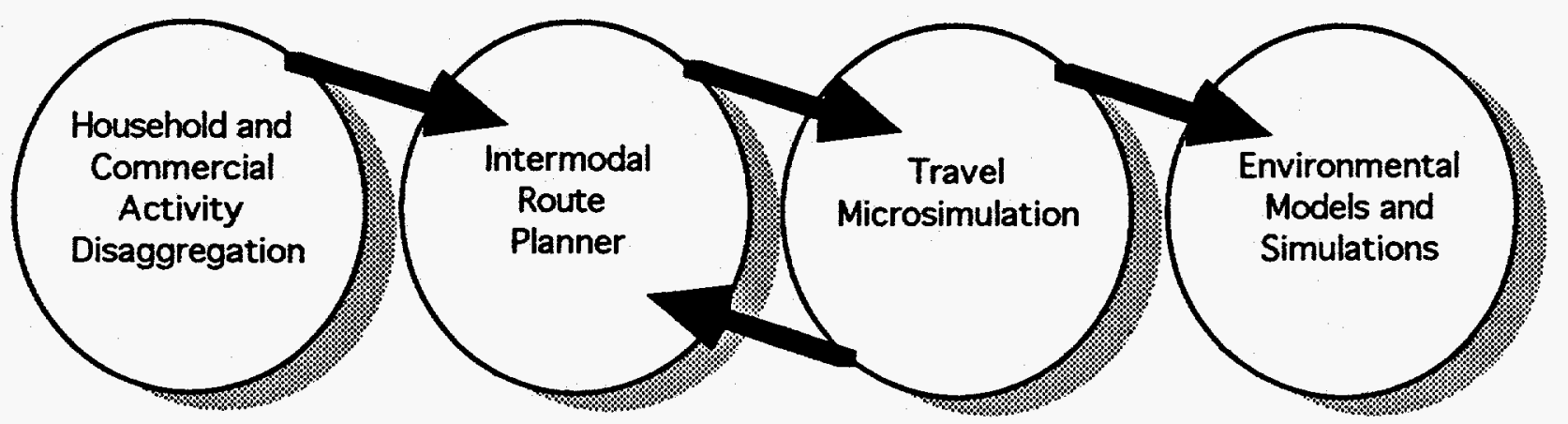


The previous figure illustrates the TRANSIMS architecture. The TRANSIMS methods deal with individual behavioral units and proceed through several steps to estimate travel. TRANSIMS predicts trips for individual households, residents, freight loads, and vehicles rather than for zonal aggregations of households. The Household and Commercial Activity Disggregation Module creates regional synthetic populations from census and other data. Using activity-based methods and other techniques, it produces a travel representation of each household and traveler. These two submodules, Synthetic Populations and Activity Demand and Travel Behavior, are described separately in the following pages.

The Intermodal Route Planner involves using a demographically defined travel cost decision model particular to each traveler. Vehicle and mode availability are represented and mode choice decisions are made during route plan generation. The method estimates desired trips not made, induced travel, and peak load spreading. This allows evaluation of different transportation control measures and travel demand measures on trip planning behaviors.

The Travel Microsimulation executes the generated trips on the transportation network to predict the performance of individual vehicles and the transportation system. It attempts to execute every individual's travel itinerary in the region. For example, every passenger vehicle has a driver whose driving logic attempts to execute the plan, accelerates or decelerates the car, or passes as appropriate in traffic on the roadway network.

The Travel Microsimulation produces traffic information for the Environmental Models and Simulations to estimate motor vehicle fuel use, emissions, dispersion, transport, air chemistry, meteorology, visibility, and resultant air quality. The emissions model accounts for both moving and stationary vehicles. The regional meteorological model for atmospheric circulation is supplemented by a model for local effects. The dispersion model is used for directly emitted contaminants and handles both local and urban scale problems. The air chemistry model includes dispersion, but is designed to deal with secondary pollutant production on larger scales.

The following pages describe each TRANSIMS module in greater detail. The last page describes our interim operational capability approach to TRANSIMS development.

\section{Synthetic Populations}

\section{Purpose}

The Synthetic Populations submodule creates a regional population imitation whose demographics closely match that of the real population. The imitation's households also are distributed spatially to approximate the regional population distribution. The synthetic population's demographics are provided to the Activity Demand submodule to derive individual and household activities requiring travel. The housedhold locations determine travel origins and destinations.

\section{Background}

The underlying TRANSIMS theme is that individual behavior and their interactions, as constrained by the transportation system, generate the transportation system's performance. To effect that performance in a 
simulation, individual behavior must be modeled. The Synthetic Populations submodule begins the process of creating those "individual" models.

\section{Approach}

The 1990 census data, including the Census Standard Tape File 3 (STF-3) and the Public Use Microdata Sample (PUMS), are used to develop a baseline population. We create distinct households for each census tract or block group area (we use census tracts, but the same methods could construct populations for block group areas). The procedure involves four stages. First, for the census tract in question the census summary tables from STF-3 and the corresponding PUMS sample are grouped by family and non-family households. Second, for each household type, we construct a multiway table of all demographics available from STF-3. In the third step we create households by random selection (according to the constructed multiway table probabilities) of similar households in the PUMS sample. The last stage ages the population to the desired date. A more detailed procedure follows for family households. The procedure is similar for nonfamily households and group quarters.

The STF-3 demographic summary tables for family households are (1) householder race by household class (a combination of household type and presence and age of children), (2) householder age, (3) family income, and (4) the number of workers in the family. We use these four summary tables and the corresponding PUMS sample to create the five-dimensional multiway table of probabilities for each combination of the five demographic variables. The methods used to construct the multiway table include iterative proportional fitting, maximum likelihood and minimum chi-square. Because the PUMS is a sample from multiple census tracts, iterative proportional fitting is not a statistically correct procedure for this construction. However, preliminary studies indicate that this procedure performs rather well and may be more practical than trying to use either maximum likelihood or minimum chi-square, each of which require optimizing hundreds of parameters.

The multiway table constructed for the five family household demographics is sparse. For example, the table for census tract 1.07 from Albuquerque, NM has 5880 cells but the corresponding PUMS contains only 2213 families. Therefore most constructed multiway table cells contain zero counts and hence zero probabilities. Small probabilities, representing a fractional count, could replace these zero probabilities and may improve the results. Additionally, demographics other than the five in the constructed table, such as the number of persons in each household, could be imputed from the PUMS. Imputation of additional demographic variables should be investigated to determine if the resulting population of family households better mimics that of the census tract.

Two options exist to select the number of families for each multiway table category. First, the actual number of families in the census tract may be multiplied by the table cell probabilities and rounded to determine the number of families with each demographic combination. Or, the families may be drawn at random according to the multiway table probabilities.

For the last stage, we are collaborating with Professor R. Kitamura to age the population.

We must develop methods to validate and verify these household construction procedures. We can check the constructed population of households with existing census data. For example, STF-3 contains a summary table for the total number of persons in family households. Because the total number of people is not 
controlled in the construction of family households, we can use this summary as one validation of the resulting population. For validation and verification of household characteristics that are not in STF-3 (for example the number of vehicles by the number of people in the households), we can construct a synthetic collection of "census tracts" and corresponding synthetic "PUMS" samples by considering PUMS samples as "census tracts" and combining approximately 20 neighboring PUMS samples. The resulting population of approximately 100,000 people then is sampled to create the "PUMS" for the constructed "census tracts".

\section{Activity Demand and Travel Behavior}

\section{Purpose}

The purpose of the Household and Commercial Activity Disaggregation's Activity Demand submodule is to generate household activities, activity priorities, activity locations, activity times, and mode and travel preferences. The activities and preferences are functions of the household demographics created by the Synthetic Population submodule. The Intermodal Route Planner uses the activities and preferences to determine individual's and load's trip plans for the region.

\section{Background}

Households and businesses have activities that must be, or are desired to be, performed during the day. Many of these activities require the transportation system to move a load (individual or freight) to a certain place at a certain time. Thus, activity demand generates travel demand. These activities and how they are performed depend on the demographics of the household and its individuals, or on the nature of the business.

At this time we are concentrating on traveler activities; modelling commercial load activities will be difficult until more data is available on shipping. Traveler activities are aggregated at a low level into household activities. Household activities then are estimated from probability distributions dependent on the household demographics. These demographics include the ages of the inhabitants, the household family type (single, married couple, married couple with small children, married couple with oider children, etc), the household income, the number of cars on the household, and the members of the household who can drive. These demographics are produced by the Synthetic Populations submodule.

\section{Approach}

Models will be developed for the generation of activities from household demographics. There is a considerable literature pertaining to such models, and we will use the existing research findings as much as possible. Similarly, models will be developed for generating mode preferences from individual demographics, again using existing research where appropriate. Local surveys will be necessary to calibrate these models.

Based on the input demographics, a list of travel activities will be produced for each household. These activities will be designated as "household" or "individual" activities, the distinction being that "individual" activities require the participation of a specific person in the household (for example, going to a workplace), whereas "household" activities may be divided or shared among the people in the household (for example, going shopping). Associated with each activity is a set of parameters defining the activity importance, the activity duration, and a time interval during which the activity must be performed, if it 
is performed at all (for example, work is mandatory, so a work trip must be made, but a shopping trip is typically not as important and may be skipped on a given day if the scheduling is too difficult). Locations, such as the household address and the workplace and school addresses, will be provided for mandatory activities. Locations of other activities (shopping) are not specified; the planner will choose these from a list for the locality.

We also will model travel behavior, that is, the propensity of individuals or households with given demographic characteristics to exhibit certain travel patterns or behavior. Travel behavior is more global than is travel demand. Hence we may use models given in the literature that are based on global data sets such as the National Personal Transportation Survey. For example, the choice of driver in a family household given the household's socioeconomic characteristics may be similar (in a probabilistic sense) across all sections of the country. At a minimum, we will model mode preferences for every individual and the travel characteristics in a family household. In particular, we need to model carpooling and travel arrangements (for example, which person shops) within family households. We will consider models of car availability rather than ownership.

Although extremely difficult to accomplish because of insufficient data, we must develop additional activity demand models for non-passenger trips, including freight, service, and commercial trips.

All models will be probabilistic in nature in that for a given set of demographics, a distribution of activities will be produced. The actual activities passed to the planner will be chosen by randomly selecting from this distribution. Logistic regression, classification and regression trees and neural nets are examples of tools that will produce these distributions.

\section{Intermodal Route Planner}

\section{Purpose}

The intermodal Route Planner generates regional individual activity-based travel demand.

\section{Background}

A "load" is a traveler or a commodity. A trip plan is a sequence of modes, routes, and planned departure and arrival times at the origin, destination(s), and mode changing facilities projected to move the load to its activity locations. We assume that travel demand derives from a load's desire or need to perform activities. The HCAD provides the Planner with disaggregated activity demand and travel behavior. The Planner assigns activities, modes, and routes to individual loads in the form of trip plans. The individual trip plans are input to the Travel Microsimulation for its analysis.

Trip plan selection is related directly to a load's desire to satisfy individual (or in the case of freight, corporate) goals. Goals measure a trip plan's acceptability and depend on the load's socioeconomic attributes and trip purpose. Typical goals include cost, time, and distance minimization, and safety and security maximization. The load's objective is to minimize the deviations from these goals.

Mode and route preferences also are important in the Planner. A preference is the inherent partiality or bias a load has for a particular mode or route. Typical preferences include departure time, origin-destination directedness, and 
congestion avoidance. These preferences reduce the Planner's (activity, mode, and route) solution space and offer significant computational savings.

\section{Approach}

The travel demand problem is formulated as a mathematical program based on a multi-goal objective function. The Planner's solution method has four phases as shown in the figure. In the first three phases, performed independently for each load, the individual's travel behavior preferences are adjusted iteratively to satisfy the travel goals. After every load has a feasible, or reasonable, trip plan, the fourth phase superimposes all trip plans on one another in space and time. The network characteristics then are updated based upon the projected interaction of all trip plans. The method then returns to the individual load trip planning phases and the entire process is repeated. The iterative process terminates when either all trip plans are feasible or after some criteria are satisfied.

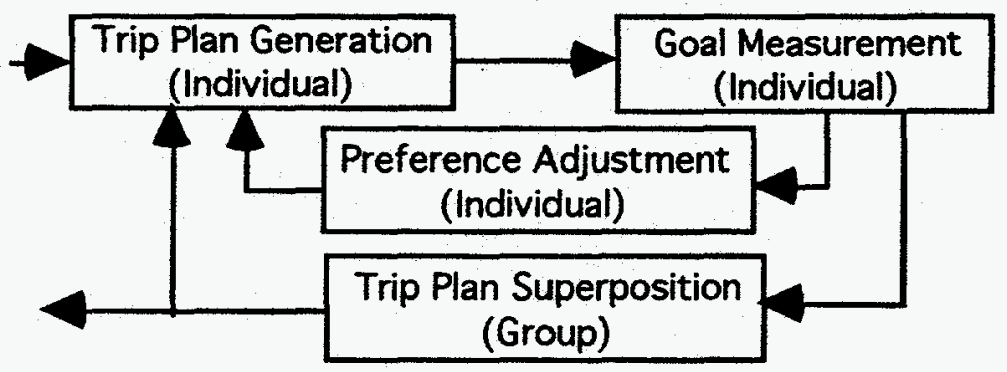

The trip plans are evaluated with respect to the individual's travel goals. For some loads, all travel goals are satisfied. For others, the plans may not satisfy some or any of the individual's travel goals. In these instances, those plans that minimize the goal deviations will be retained. The result will be trip plans that represent regional travel demand and its activity, mode, and route choice variability.

The Planner accounts for latent travel demand by travel goal deviations and unplannable activities. Goal deviations measure the load's dissatisfaction with the transportation system. If one must travel longer than desired to get to work, the deviation from one's goal travel time represents an unsatisfied demand. If a transportation infrastructure change is considered, its value can be measured by the reduction in the population's travel goal deviations. Unplannable activities are those that the load must forego because of the transportation system's deficiencies. The Planner will attempt to route the load's low priority activities after all high priority activities have been scheduled. These activities will be planned only if the current and subsequent high priority trip plans are feasible. The second latent demand measure is these unplannable low priority activities. Thus, a measure of effectiveness of any proposed facility change is the change in unplannable activities and the resulting trip plans.Loads plan their trips based on activity requirements, knowledge of the transportation system, travel goals, and assumptions about other load trip plans. The travel-planning decisionmaking process can be achieved iteratively with the Planner and Microsimulation. Feedback loops between both modules mimic individual's real travel process of plan, execute, and replan.

The Intermodal Route Planner model generates activity-based travel demand at the individual load level. By (1) receiving activity demand and travel behavior from the HCAD, (2) providing individual trip plans to the Travel 
Microsimulation, and (3) obtaining feedback from the Microsimulation from the trip plan execution, the Planner forecasts regional travel demand over multiple time scales.

\section{Travel Microsimulation}

\section{Purpose}

The TRANSIMS Travel Microsimulation module mimics the movement and interactions of travelers thoughout a metropolitan region's transportation system. For this discussion, traveler refers both to human travelers as well as freight loads, etc. The Intermodal Route Planner provides a trip plan to each traveler that he then attempts to execute on the transportation network. In the process he interacts with other travelers and the transportation system. The combined traveler interactions produce emergent behaviors such as traffic congestion.

\section{Background}

The TRANSIMS Travel Microsimulation models many transportation modes including automobiles, trucks, buses, light rail, commuter rail, bicycles, and pedestrians. Thus, the microsimulation includes roadway, transit, rail, bikeway, and pedestrian networks. In the following discussion, we illustrate the TRANSIMS microsimulation with roadway transportation examples because of its high use, complexity, and importance to air quality. The roadway network includes freeways, highways, streets, ramps, turn lanes, grades, and intersections (signalized or unsignalized). In executing their trip plans, vehicle drivers accelerate, decelerate, turn, change lanes, pass, and respond to other vehicles and signs and signals. Drivers exhibit behavior between aggressive and passive. Vehicles have weight and acceleration and deceleration characteristics. Analysis requirements determine the necessary microsimulation detail.

Increasing the microsimulation's detail increases its behavioral representation of real transportation systems, but it also increases its computational burden. The representation quality is called the model's fidelity. One goal is to find the minimum computational detail necessary to produce the fidelity needed for specific analyses. This minimum computational detail is called critical complexity. A hybrid technique uses high-fidelity microsimulations for areas where detailed results are needed and low-fidelity, fast-running microsimulations for areas where there is less interest. This hybrid microsimulation requires matching the microsimulations at their boundaries.

\section{Approach}

We are studying two approaches to the microsimulation. Applications and investigations with the two approaches will form the basis for deciding which approach will be used in later TRANSIMS versions.

In the first approach, the links (roadway segments) of the network representation of the transportation system are a continuous domain. A vehicle can be positioned along any point on the segment. The vehicle driver evaluates the current situation and decides his next action that advances the vehicle to a new position. The vehicle and driver objects retain their characteristics as they move through the network.

The second approach is to use a cellular automata (CA) microsimulation. CA traffic models divide the transportation network into a finite number of cells. For example, each cell could be approximately the length of a vehicle. At each 
time step of the simulation, each cell is examined for a vehicle occupant. If a vehicle is present in the cell, the vehicle is advanced to another cell according to a simple rule set. A CA microsimulation is low fidelity, but provides a means to simulate large numbers of vehicles and maintain a fast execution speed. Increasing the fidelity by decreasing the cell size, adding vehicle attributes, and expanding the rule set results in slower computational speed. We will explore the fidelity and performance limits of the CA microsimulation to establish the computational detail necessary to meet the analysis requirements.

The primary Travel Microsimulation output is the second-by-second location of each traveler. Analysis of the primary output yields additional information such as velocities, accelerations, decelerations, average speeds, average travel times. Plots such as travel time vs traffic density, traffic flow vs traffic density, vehicle positions vs time; and animation such as vehicle movements on network segments also can be generated. Data on positions, velocities, accelerations, decelerations, and vehicle total travel time also is input to the emissions model to determine effluents at spatial locations thoughout the region.

\section{Environmental Models and Simulation}

\section{Purpose}

The purpose of the environmental module is to translate traveler behavior into consequent air quality, energy consumption, and carbon dioxide emissions. The environmental module will use information from the planner and the microsimulation and it will support the analyst's toolbox. It also could provide information on fog to the microsimulation.

\section{Background}

Transportation systems play a significant role in urban air quality, energy consumption and carbon-dioxide emissions. Recently, it has been found that current systems for estimating emissions of pollutants from transportation devices lead to significant inaccuracies. When these inaccuracies are coupled to air quality models and limited meteorological data, it is difficult to tell whether the most appropriate path is being taken to achieve air quality goals. Most existing emission modules use very aggregate representations of traveler behavior and attempt to estimate emissions on typical driving cycles. However, recent data suggests that typical driving cycles produce relatively low emissions with most emissions coming from off-cycle driving, cold-starts, and evaporative emissions. Furthermore, some portions of the off-cycle driving such as climbing steep grades are apt to be correlated with major meteorological features such as downslope winds. These linkages are important, but they are not treated systematically in the current modeling systems.

\section{Approach}

We plan to develop a system of linked modules including: (1) emissions modules, (2) regional-scale meteorological models, (3) microscale meteorological modules (street-canyon), (4) dispersion and transport modules, and (5) air chemistry (airshed) modules. The development of these modules will build upon efforts already underway at Los Alamos and the larger air quality community. At Los Alamos we have experience with regional scale (urban metropolitan area) meteorological models that can describe airflow and turbulence driven by terrain and land use without the requirement of many local measurements. We have dispersion and transport models that can take the information from the meteorological model and describe the dispersion that occurs in complex terrain. 
We also have available an airshed model (through collaboration with investigators at Carnegie Mellon University) that uses the meteorologicalmodule outputs with emissions to describe the air-chemistry in a metropolitan area. We currently are developing an appropriate emissions model and a simplified microscale meteorological module.

The emissions module must take the information on individual traveler behavior and produce NOx, CO, aerosol, and hydrocarbon emissions for input to both the airshed and dispersion models. We will have individual vehicle motion available on one-second intervals from the Travel Microsimulation. We also will have traveler's plans that describe when vehicles are used and when and where they are stationary. In our preliminary emissions module we will integrate an existing modular emissions model, VEHSIME, with existing models for evaporative emissions, cold-start emissions, and high-emitting vehicle emissions. We also will develop a preliminary micro-scale meteorological model based on adding street-canyon eddies to our dispersion module. At the same time we are adding a capability to treat fog and clouds to our meteorological model.

In the longer term the preliminary emissions module will be replaced by a physics and chemistry-based model being developed at the University of Michigan with additional modifications being developed by other studies. We also will develop or acquire an aerosol emissions model to be used for both diesel and gasoline engines. The air chemistry model will be extended to address organic aerosol production.

\section{Interim Operational Capabilities}

We visited six metropolitan planning organizations (MPOs) (Dallas-Ft. Worth, Boston, Portland OR, Oakland, Chicago, and Denver). We presented the overall TRANSIMS approach and obtained information on their responsibilities, transportation and air quality issues, processes for carrying out their activities, potential applications for TRANSIMS, their resources, and user feedback on what TRANSIMS should do for them. We are using this and other information to develop detailed requirements and specifications for the TRANSIMS architecture and design.

To provide greater, more timely interaction and feedback from the TRANSIMS user community, we have formulated an approach for TRANSIMS development in which we will develop an interim operational capability (IOC) for each major TRANSIMS module during the five-year program. When the $1 O C$ is ready, we will complete a specific case study to confirm the $10 \mathrm{C}$ features, applicability, and readiness. We will complete the specific case study with the collaboration of the staff of a selected MPO. This approach should give us quicker feedback from the user community and provide interim products, capabilities, and applications. This approach maintains our goal of an integrated framework for predicting individual travel behavior and for supporting transportation planners from travel demand forecasting to assessments of transportation system modifications.

The Travel Microsimulation will be the first IOC, with the goal of having it ready for testing in August 1995. As this $10 \mathrm{C}$ is developed, we will work with the selected MPO to identify studies that the IOC should support. The second IOC will integrate the air quality analysis capability of TRANSIMS with the Travel Microsimulation. Again, the $10 C$ development will be driven by studies identified as important to the users and will be followed by the specific case studies. We intend to issue subsequent $10 \mathrm{Cs}$ for the Intermodal Route Planner and for the Household and Commercial Activity Disaggregation modules. These IOCs may be 
standalone modules, but will be capable of integration with the other TRANSIMS modules. The case studies will demonstrate the integrated package.

We have developed several microsimulation versions that successfully modeled traffic behavior. In the Albuquerque Demonstration, we simulated traffic on two interstates and at their intersection using vehicle objects on a continuous road network. For the IVHS Incident Detection Testbed, we extended this capability to include many lanes, signalized intersections, incidents, and additional driver behavior. Our single-lane cellular automata (CA) simulation exhibited traffic congestion, shock waves, and roadway capacity. An enhanced $C A$ version runs on distributed processers and dynamically redistributes the computational load during execution. Another CA version with multiple lanes and freeway interchanges simulated critical traffic volumes on the $48,000 \mathrm{~km}$ of the entire German Autobahn using a 64-node partition of the Intel Paragon parallel processor machine. This broad microsimulation experience places us in an excellent position to take the best of what we have learned to develop the first Travel Microsimulation IOC.

To the extent possible the first Travel Microsimulation $10 \mathrm{C}$ will rely on data currently existing at MPOs. We will develop techniques to incorporate these data readily into the TRANSIMS methodology. The IOC will be supported with the capability to let the user adjust the input as necessary for his analyses. Similarly the user will be supported with several output options to support his analyses. We will develop these features with input and feedback from the potential users.

From our MPO visits we had sufficient information to decide which regional area to use for our first case study. We considered numerous factors, but the major ones were: staff and management interest, staff capabilities, and data availability. The ratings were very close and the decision was difficult, but we decided to work with the North Central Texas Council of Governments (NCTCOG) (Dallas-Fort Worth) for the first case study using the Travel Microsimulation IOC. NCTCOG has enthusiastically supported this decision.

The input, feedback, and interest of all the MPOs we visited have been very helpful in establishing the scope and requirements for TRANSIMS. We anticipate that the future 1OCs will be developed in association with case studies for other metropolitan regions. We also anticipate that there may be other supporting developmental efforts in which collaboration with a metropolitan region would be helpful both to the TRANSIMS effort and the MPO.

\section{Acknowledgements}

Although the authors accept responsibility for the TRANSIMS descriptions in this document, there are numerous others who have contributed, or are contributing, to the TRANSIMS Project. Without their support, ideas, and input, this document would not be possible. Chris Barrett and Darrell Morgeson share responsibility for the TRANSIMS vision and initiative. Other contributors include: Kathy Berkbigler, Mike Brown, Brian Bush, John Davis, Deborah Kubicek, Verne Loose, Mike McKay, Jack Morrison, Kai Nagel, Rob Oakes, Steen Rasmussen, Marcus Rickert, Jay Riordan, Doug Roberts, Paula Stretz, Steve Sydoriak, Gary Thayer, and Murray Wolinsky. We also appreciate the support and feedback of our sponsors at the Federal Highways Administration, the Department of Transportation, and the Environmental Protection Agency: Fred Ducca, Ed Weiner, Ron Jensen-Fisher, Ron Giguerre, and Mark Simons. 